\title{
Association between Muscle Mass and Body Mass Index in Elderly Diabetic Patients Attending Tertiary Care Center in Bangalore, India
}

\author{
Sowbarnika Palanisami, ${ }^{1}$ Vasudha Kulkarni. ${ }^{7}$
}

\begin{abstract}
Background: Sarcopenia is a disorder causing age-related loss of muscle mass. Its multifaceted nature has been linked to an increased risk of disability and mortality. Equally, obesity is a well-known risk factor for a host of disorders. A combination of sarcopenia and obesity in elderly diabetics can synergistically lead to increased insulin resistance and risk of metabolic syndrome. This study aimed to identify the association between sarcopenia and obesity in elderly diabetic patients by a cost-effective anthropometric method. Methods: A case-control study was conducted from January 2016 to April 2016 at Dr. B. R. Ambedkar Medical College in Bangalore. Height, weight, mid-arm circumference, and triceps skin fold thickness of 112 diabetic patients and 131 healthy adults were measured. Descriptive statistical analysis and multiple linear regression analysis were carried out. Results: $26.8 \%$ of male and $76.8 \%$ of female diabetic patients were obese (body mass index $\geq 25 \mathrm{~kg} / \mathrm{m}^{2}$ ). Incidence of sarcopenia (muscle mass one standard deviation smaller than healthy reference population, cut-off value for diabetic males being $<9.79 \mathrm{~kg} / \mathrm{m}^{2}$ and for diabetic females $<8.53 \mathrm{~kg} / \mathrm{m}^{2}$ ) were $12.5 \%$ in male diabetic patients and $5.4 \%$ in female diabetic patients. Conclusion: Sarcopenia and obesity are co-morbid illnesses which can cause functional and metabolic impairments in elderly diabetic patients. There exists a moderate association between muscle mass and body mass index. Loss of muscle strength (dynapenia), rather than loss of muscle mass (sarcopenia), is closely associated with disabilities in these patients.
\end{abstract}

Keywords: Anthropometry; Sarcopenia; Obesity; Diabetes Mellitus; Body Mass Index (Source: MeSH-NLM).

About the Author: Sowbarnika Palanisami is a Phase III medical student at Dr. B.R. Ambedkar Medical College in Bangalore, India.

\section{Introduction}

"Successful ageing"-a capacity to maintain quality of life, health, and independence in later years of life-is a challenging issue. In order to minimize disability and chronic ailments, it is imperative to identify lifestyle, health, and social factors that would impact functional ability of elderly individuals.' One of the most common health disorders affecting elderly individuals is diabetes mellitus. Diabetes and its complications take a major toll on the quality of life in elderly patients, with increased risk of cardiovascular mortality and health care costs of the society. ${ }^{2}$ In 2015, 415 million people (male: 215.2 million, female: 199.5 million) aged 20-79 years were diabetic according to International Diabetic Federation (IDF) (Available from: http:// www.diabetesatlas.org/component/attachments/?task=downloadctid=116, updated 2015; cited 2016 Aug 20). In 2007, India had the largest number of diabetics, at about 40.9 million ( $15 \%$ of the global diabetics). ${ }^{2}$ This number is expected to go up to 69.9 million by 2025 (Available from: http://clinicalestablishments.nic.in/WriteReadData/58.pdf, updated 2008; cited 2016 Aug 20).

Most striking changes in elderly diabetic patients are obesity and decreased skeletal muscle mass.' Reduction in skeletal muscle mass is termed "sarcopenia", which comes from Greek words "sarx", for flesh, and "penia", for loss. ${ }^{3}$ The molecular basis for the effects of sarcopenia is that skeletal muscle is one of the primary sites for glucose uptake and storage, and myokines secreted by skeletal muscle counteract the metabolic and pro-inflammatory effects of adipocytes. The combined effect of sarcopenia and obesity can increase the risk of developing metabolic and cardiovascular diseases. ${ }^{4}$

Many methods are available for the evaluation of sarcopenia, such as computed tomography, magnetic resonance imaging, dual energy $\mathrm{X}$-ray absorptiometry, prediction using total body potassium, and creatinine excretion. These methods are expensive, and anthropometry is a cost-effective alternative method to estimate sarcopenia and obesity with considerable accuracy. ${ }^{5} \mathrm{~A}$ previous study comparing these methods has proven anthropometry to be an easy and inexpensive method. ${ }^{6}$ The need for the present study was to examine if an anthropometrical association exists between sarcopenia and obesity. Muscle mass and body mass indices were measured with parameters such as height, weight, corrected arm muscle area, and triceps skinfold thickness to identify the association between sarcopenia and obesity.

\section{Methods}

Ethics Statement

Written informed consent was obtained from all the partici- 
pants (elderly diabetic patients and healthy adult group). Protocol was approved by Institutional Ethical Committee of Dr. B.R. Ambedkar Medical College, Bangalore prior to initiation of the study. The study procedures were carried out in accordance with the principles of Declaration of Helsinki.

\section{Study Design and Population}

An observational, case-control study was carried out from February 2016 to March 2016. Pilot study was done on 21 elderly diabetic patients and 25 normal subjects to determine the sample size. The sample included 112 diabetic patients ( 56 males and 56 females) aged $\geq 60$ years with an existing diagnosis of type 1 or type 2 diabetes mellitus. To estimate cut off values of sarcopenia (which is defined as muscle mass one standard deviation less than those of normal young subjects), 131 normal subjects ( 56 males and 75 females) aged 17 to 58 years were considered. Age-matched control groups were not considered for this study.

Diabetic patients with history of renal disorder, liver disorder, cancer, organ failure and those who had previously undergone limb amputation were excluded. In normal subjects, history of diabetes, stroke, coronary artery disease, cancer, liver cirrhosis, hypertension, and organ failure were ruled out. In both the case and control groups, history of smoking and alcohol consumption were elicited, and individuals with such history were excluded.

Based on the history of diabetes and age, the participants were stratified into case (i.e. those with diabetes and age $>60$ ) and control groups (i.e. those without history of diabetes and aged <60), taking into consideration the exclusion criteria for both groups.

\section{Anthropometric Measurements and Statistical Analysis}

Anthropometric measurements of height, weight, mid-arm circumference and triceps skin fold thickness were taken based on the following methods: (1) weight was measured to the nearest $0.1 \mathrm{~kg}$ in light clothes without footwear, using a digital scale; (2) standing height was measured using a height scale fixed to the wall and was measured to the nearest $0.1 \mathrm{~cm}$; (3) a point midway between the tip of acromion process of scapula and olecranon process was marked with the participant's arm flexed at $90^{\circ}$, and this point was used to calculate mid-arm circumference to the nearest $0.1 \mathrm{~cm}$; and (4) triceps skin fold thickness was measured over the same point on the posterior aspect of arm over the triceps muscle using a Holtain caliper to the nearest $0.1 \mathrm{~cm}$.?

Body mass index was calculated by dividing weight $(\mathrm{kg})$ with the square of height (in meters), and obesity was defined as body mass index $\geq 25 \mathrm{~kg} / \mathrm{m}^{2}$ in our study population. ${ }^{8}$ The midarm circumference (MAC) and triceps skin fold thickness (TSF) were used to calculate the corrected arm muscle area (CAMA) using the formula: CAMA $=[\mathrm{MAC}-(\pi \times \mathrm{TSF})]^{2} / 4 \pi-\mathrm{BA}$ (BA is correction for bone area, which is $10 \mathrm{~cm}^{2}$ for males and $6.5 \mathrm{~cm}^{2}$ for females). ${ }^{6}$ Muscle mass was calculated with corrected arm muscle area and triceps skin fold thickness using the formula: muscle mass $(\mathrm{kg})=$ height $\times(0.0264+0.0029 \times$ CAMA $){ }^{6}$

Sarcopenia was defined as muscle mass more than one standard deviation below the mean of the young reference population (cut off values were $49.79 \mathrm{~kg} / \mathrm{m}^{2}$ for diabetic males and $<8.53 \mathrm{~kg} / \mathrm{m}^{2}$ for diabetic females). SPSS software version 21 was used for statistical analysis. Descriptive statistical analysis was done to calculate geometric means and standard deviations for variables such as age, body height, body weight, body mass index, mid-arm circumference, triceps skin fold thickness, corrected arm muscle area and muscle mass of normal and elderly diabetic patients. Multiple linear regression analysis was done to obtain the relationship between the dependent variables (body mass index and muscle mass) and the three independent variables age, height and weight. ANOVA test was administered to find out the breakdown of variance between groups (normal males, normal females, diabetic males and diabetic females) in parameters like muscle mass and body mass index.

\section{Results}

131 non-diabetic participants and 112 elderly diabetic patients contributed to the anthropometric data. The mean (with standard deviation) age, height, weight, body mass index, mid-arm circumference, triceps skin fold thickness, corrected arm muscle area and muscle mass for both the case and control groups are presented in Table 1.

Out of 112 elderly diabetic patients, $8.9 \%$ patients had sarcopenia (male: $12.5 \%$; female: $5.4 \%$ ). The prevalence of obesity among elderly diabetic patients were $26.8 \%$ for males and $76.8 \%$ for fema-

Table 1. Characteristics of Normal Participants and Elderly Diabetic Patients at $95 \%$ Confidence Interval

\begin{tabular}{|c|c|c|c|c|}
\hline Characteristics & \multicolumn{2}{|c|}{ Male } & \multicolumn{2}{|c|}{ Female } \\
\hline Age (years) & $29.8 \pm 1.4$ & $66.2 \pm 1.1$ & $29.4 \pm 1.2$ & $64.8 \pm 0.8$ \\
\hline Body height (m) & $1.72 \pm 0.01$ & $1.66 \pm 0.01$ & $1.57 \pm 0.01$ & $1.50 \pm 0.01$ \\
\hline Body mass index, $\mathrm{kg} / \mathrm{m}^{2}$ & $23.28 \pm 0.59$ & $25.23 \pm 0.64$ & $23.76 \pm 0.66$ & $28.80 \pm 0.76$ \\
\hline Mid-arm circumference $(\mathrm{cm})$ & $29.1 \pm 0.5$ & $28.0 \pm 0.5$ & $27.1 \pm 0.5$ & $28.3 \pm 0.5$ \\
\hline Triceps skinfold thickness $(\mathrm{cm})$ & $3.5 \pm 0.1$ & $2.5 \pm 0.1$ & $3.2 \pm 0.1$ & $2.9 \pm 0.1$ \\
\hline Corrected arm muscle area $\left(\mathrm{cm}^{2}\right)$ & $16.95 \pm 0.83$ & $23.28 \pm 1.34$ & $17.55 \pm 0.91$ & $23.36 \pm 1.29$ \\
\hline
\end{tabular}

Values are means \pm standard deviations. 
les. The correlation coefficients between muscle mass and body mass index were 0.38 in male diabetic patients and 0.58 in female diabetic patients.

Multiple linear regression analysis was performed to identify predictors of muscle mass, with muscle mass being the dependent variable and predictors being age, height and weight. The $R^{2}$ value indicated that $67 \%$ of the variance in muscle mass was explained by variance in the three predictor variables. $\beta$ values indicated that weight had greater influence on muscle mass $(\beta=0.605)$ than age $(\beta=0.216)$.

\section{Discussion}

We compared the association of muscle mass and body mass index in elderly diabetic patients (male and female patients aged $\geq 60$ years) with normal participants aged $17-58$ years who had no history of diabetes mellitus. In normoglycemic individuals, corrected arm muscle area and triceps skin fold thickness correlated positively with body mass index, suggestive of their role in the magnitude of body mass index. In elderly diabetic individuals, triceps skin fold thickness was more closely related to body mass index than muscle mass. We found an average association between obesity and sarcopenia in the participants with diabetes mellitus.

Skeletal muscle composition estimated using upper arm anthropometry is a key diagnostic method for identifying geriatric syndromes associated with sarcopenia.? Among various anthropometric methods available to determine sarcopenia, a simple formula "fat-free muscle mass index = fat-free mass $(\mathrm{kg}) /$ height ${ }^{2}\left(\mathrm{~m}^{2}\right)^{\prime \prime}$ was proposed in 2013 , with sarcopenia defined as two standard deviations less than young adult reference population. ${ }^{9}$ With dual energy X-ray absorptiometric technique, muscle mass can be estimated by the formula "skeletal muscle mass index = appendicular skeletal muscle mass $(\mathrm{kg}) / \mathrm{height}^{2}$ $\left(\mathrm{m}^{2}\right)^{\prime \prime}$, with sarcopenia defined as values lower than $7.36 \mathrm{~kg} /$ $\mathrm{m}^{2}$ for men and $5.81 \mathrm{~kg} / \mathrm{m}^{2}$ for women. ${ }^{10}$ Based on the fact that sarcopenia and obesity act synergistically on metabolic and functional impairments in elderly, the formula "appendicular muscle mass/body weight" was used to define sarcopenic obesity. ${ }^{11,12}$ However, Bret in 1997 proposed that upper body fat distribution heightens the risk for insulin resistance and metabolic syndrome. ${ }^{13}$ This is the basis for the calculation of muscle mass using Heymsfield formula with parameters such as corrected arm muscle area and triceps skin fold thickness in the present study. ${ }^{6}$

The possible association of muscle mass and body mass index can be explained by their interaction at molecular level. Age-related muscle wasting leads to reduced physical activity and induces accumulation of visceral fat. Increase in abdominal fat particularly may release pro-inflammatory cytokines, tumor necrosis factor- $\alpha$ (TNF- $\alpha$ ) and leptin. This causes fatty infiltration in the muscle tissue and contributes to loss of muscle mass. Thus, obesity can hasten the process of reduction of muscle mass. ${ }^{14}$ In earlier studies, leg muscle mass has been considered to be an important determinant of central arterial stiffness. Increase in the muscle mass can increase the requirement of blood supply resulting in higher cardiac output and stroke volume. This can contribute to larger size adaptation of arteries.? Insulin resistance in diabetic individuals with sarcopenia can be attributed to greater lipolytic activity of centrally located adipocytes. Portal venous elevation of fatty acids decreases the hepatic insulin extraction and promotes the synthesis of apolipoprotein-B and lipoproteins. Obesity and sarcopenia can thus result in hyperinsulinemia. ${ }^{9}$ A cross-sectional study of lean young Indian men showed that muscle metabolism significantly correlated with glucose disposal rate. This was suggestive of the fact that muscle is an important location for glucose disposal and therefore insulin sensitivity. ${ }^{15}$

A study in Korean population conducted in 2009-2010 has shown that sarcopenia may be an early predictor for insulin resistance, diabetes mellitus and metabolic syndrome. ${ }^{16} \mathrm{~A}$ similar study conducted among elderly Korean adults in 2008-2010 revealed that there was a strong association between hypertension and sarcopenia. ${ }^{16}$ The risk of hypertension was found to be four-fold higher in diabetic patients with sarcopenic obesity. Note, however, that heavy alcohol consumption and smoking had to be taken into account while assessing the relationship between sarcopenia and hypertension." In addition, a Japanese study has proven that physical activity of moderate intensity ( $>15-20$ minutes/day) maintained muscle mass beyond sarcopenia threshold.'

In the present study, muscle mass showed an average relationship with body mass index in diabetic patients. Several prior studies have indicated that lower muscle mass has a greater influence on insulin sensitivity in diabetic patients than fat distribution. ${ }^{17-25}$ In addition, earlier studies on the combined effects of obesity and lower muscle mass in older persons on physical functioning have suggested that older patients with sarcopenic obesity had two-fold greater risk of developing instrumental activities of daily living disability than those without sarcopenic obesity. ${ }^{1}$

Bedside estimation of muscle mass by anthropometry has proven to be of prognostic and therapeutic value. A screening method using anthropometric prediction equation can be a cost-effective diagnostic tool for determining sarcopenia in elderly individuals in primary health care settings. Such screening programs followed by confirmatory diagnosis of sarcopenia with dual energy $\mathrm{X}$-ray absorptiometry would be beneficial to support early treatment of diabetes mellitus. ${ }^{26}$

Cautions must be taken in the assessment of muscle mass, as anthropometrical muscle mass could change independently from muscle composition, especially in cases of protein energy malnutrition. In severe protein energy malnutrition, the water content increase per gram of wet muscle tissue, masking to some extent losses of muscle functional protein. ${ }^{6}$

The present study provides baseline data of muscle mass of community-based elderly diabetic population in Bangalore, India. The accuracy and reliability of the findings are limited by the potential errors in the anthropometric measurements. Highly trained observers and frequent measurements are required for diagnostic accuracy, for there could be individual variability in the measurement of triceps skin fold thickness and mid-arm circumference area. There is also a need to develop a non-invasive anthropometric method to determine muscle mass index with desirable diagnostic accuracy which could 
serve as a screening tool for measuring muscle strength rather than muscle mass.

In conclusion, there was a positive correlation between muscle mass and body mass index in elderly diabetic patients. The prevalence of obesity in elderly diabetic patients were $76.8 \%$ and $26.8 \%$ among female and male patients, respectively. Our study concurs with earlier studies which have proven that muscle strength, rather than muscle mass, is beneficial for establishing relationship between muscle mass and body mass index. Further studies are needed to establish the relationship between muscle strength and body mass index.

\section{References}

1. Levine ME, Crimmins EM. The impact of insulin resistance and inflammation on the association between sarcopenic obesity and physical functioning. Obesity (Silver Spring). 2012 0ct;20(10):2101-6.

2. Jain A, Paranjape S. Prevalence of type 2 diabetes mellitus in elderly in a primary care facility: an ideal facility. Indian J Endocrinol Metab. 2013 Oct;17(Suppl1): S318-22.

3. Stenholm S, Harris TB, Rantanen T, Visser M, Kritchevsky SB, Ferrucci L. Sarcopenic obesity: definition, cause and consequences. Curr Opin Clin Nutr Metab Care. 2008 Nov;11(6):693-700.

4. Srikanthan P, Hevener AL, Karlamangla AS. Sarcopenia exacerbates obesity-associated insulin resistance and dysglycemia: findings from the $\mathrm{Na}$ tional Health and Nutrition Examination Survey III. PLOS One. 2010 May 26;5(5):e10805.

5. Kuriyan R, Krupad AV. Prediction of total body mass from simple anthropometric measurements in young Indian males. Indian J Med Res. 2004 Mar;119(3):121-8.

6. Heymsfield SB, McMannus C, Smith J, Stevens V, Niscon DW. Anthropometric measurement of muscle mass: revised equations for calculating bone-free arm muscle area. Am J Clin Nutr. 1982 Oct;36(4):680-90.

7. Villani AM, Crotty M, Cameron ID, Kurrle SE, Skuza PP, Cleland LG, et al. Appendicular skeletal muscle in hospitalised hip-fracture patients: development and cross-validation of anthropometric prediction equations against dual-energy X-ray absorptiometry. Age Ageing. 2014 Nov;43(6):857-62.

8. Snehalatha C, Viswanathan V, Ramachandran A. Cutoff values for normal anthropometric variables in asian Indian adults. Diabetes Care. 2003 May;26(5):1380-4.

9. McIntosh El, Smale KB, Vallis LA. Predicting fat-free mass index and sarcopenia: a pilot study in community-dwelling older adults. Age (Dordr). 2013 Dec;35(6):2423-34.

10. Yu S, Appleton S, Chapman I, Adams R, Wittert G, Visvanathan T, et al. An anthropometric prediction equation for appendicular skeletal muscle mass in combination with a measure of muscle function to screen for sarcopenia in primary and aged care. J Am Med Dir Assoc. 2015 Jan;16(1):25-30.

11. Lim S, Kim JH, Yoon JW, Kang SM, Choi SH, Park YJ, et al. Sarcopenic obesity: prevalence and association with metabolic syndrome in the Korean Longitudinal Study on Health and Aging (KLOSHA). Diabetes Care. 2010

\section{Jul;33(7):1652-4.}

12. Han K, Park YM, Kwon HS, Ko SH, Lee SH, Yim HW, et al. Sarcopenia as a determinant of blood pressure in older Koreans: findings from the Korea National Health and Nutrition Examination Surveys (KNHANES) 2008-2010. PLOS One. $2014 \operatorname{Jan}$ 29;9(1):e86902.

13. Goodpaster BH, Thaete FL, Simoneau JA, Kelley DE. Subcutaneous abdominal fat and thigh muscle composition predict insulin sensitivity independently of visceral fat. Diabetes. 1997 Oct;46(10):1579-85.

14. Ryu M, Jo J, Lee Y, Chung YS, Kim KM, Baek WC. Association of physical activity with sarcopenia and sarcopenic obesity in community-dwelling older adults: the Fourth Korea National Health and Nutrition Examination Survey. Age Ageing. 2013 Nov;42(6):734-40.

15. Unni US, Ramakrishnan C, Raj T, Kishore RP, Thomas T, Vaz M, et al. Muscle mass and functional correlates of insulin sensitivity in lean young Indian men. Eur J Clin Nutr. 2009 0ct;63(10):1206-12.

16. Moon SS. Low skeletal muscle mass is associated with insulin resistance, diabetes, and metabolic syndrome in the Korean population: the Korea National Health and Nutrition Examination Survey (KNHANES) 2009-2010. Endocr J. 2014;61(1):61-70.

17. Kim TN, Park MS, Yang SJ, Yoo HJ, Kang HJ, Song W, et al. Prevalence and determinant factors of sarcopenia in patients with type 2 diabetes: the Korean Sarcopenic Obesity Study (KSOS). Diabetes Care. 2010 Jul;33(7):1497-9. 18. Kalyani RR, Tra Y, Egan JM, Ferrucci L, Brancati F. Hyperglycemia is associated with relatively lower lean body mass in older adults. J Nutr Health Aging. 2014;18(8):737-43.

19. Wen $\mathrm{X}$, Wang $\mathrm{M}$, Jiang CM, Zhang YM. Anthropometric equation for estimation of appendicular skeletal muscle mass in Chinese adults. Asia Pac J Clin Nutr. 2011 Jul;20(4):551-6.

20. Lee JS, Auyeung TW, Leung J, Kwok T, Leung PC, Woo J. The effect of diabetes mellitus on age-associated lean mass loss in 3153 older adults. Diabet Med. 2010 Dec;27(12):1366-71.

21. Goel K, Gupta N, Misra A, Poddar P, Pandey RM, Vikram NK, et al. Predictive equations for body fat and abdominal fat With DXA and MRI as reference in Asian Indians. Obesity (Silver Spring). 2008 Feb;16(2):451-6.

22. Lee CC, Glickman SC, Dengel DR, Brown MD, Supiano MA. Abdominal adiposity assessed by dual energy $\mathrm{X}$-ray absorptiometry provides a sex-independent predictor of insulin sensitivity in older adults. J Gerontol A Biol Sci Med Sci. 2005 Jul;60(7):872-7.

23. Bijlsma AY, Meskers CG, van Heemst D, Westendorp RG, de Craen AJ, Maier $A B$. Diagnostic criteria for sarcopenia relate differently to insulin resistance. Age (Dordr). 2013 Dec;35(6):2367-75.

24. Kulkarni B, Kuper H, Taylor A, Wells JC, Radhakrishna KV, Kinra S, et al. Development and validation of anthropometric prediction equations for estimation of lean body mass and appendicular lean soft tissue in Indian men and women. J Appl Physiol (1985). 2013 Oct 15;115(8):1156-62.

25. Campbell TM, Vallis LA. Predicting fat-free mass index and sarcopenia in assisted-living older adults. Age (Dordr). 2014 Jul;36(4):9674.

26. Lee CG, Boyko EJ, Barrett-Connor E, Miljkovic I, Hoffman AR, Everson-Rose $S A$, et al. Insulin sensitizers may attenuate lean mass loss in older men with diabetes. Diabetes Care. 2011 Nov;34(11):2381-6.

\section{Acknowledgments}

We thank Dr. B. R. Ramesh, Head of Department of Anatomy, Dr. B. R. Shivakumar, Department of General Medicine, Dr. S. V. Divakar, principal and teaching faculty of Department of Anatomy and Medicine of Dr. B.R. Ambedkar Medical College and Hospital, Bangalore, India for supervision of the research work and Dr. Uma. G, Department of Statistics, PSG College of Arts and Science, Coimbatore, India for statistical assistance.

Conflict of Interest Statement a Funding

The authors have no funding, financial relationships, or conflicts of interest to disclose.

\section{Author Contributions}

Conceptualization, Data collection, Writing, Approval of the final version: SP, VK. Data analysis and interpretation, Critical revision of the manuscript, Statistical advice: VK.

Cite as:

Palanisami S, Kulkarni V. Association between muscle mass and body mass index in elderly diabetic patients attending tertiary care center in Bangalore, India. Int J Med Students. 2016 Sep-Dec;4(3):96-9. 